Cell Research (2000),10, 29-38

\title{
Molecular characters and morphological genetics of CAL gene in Chinese cabbage*
}

\author{
Li Xiao Fang, Rui Juan Shen, Ping Lin Liu, Zhang Cheng \\ TANG, Yu Ke HE** \\ Shanghai Institute of Plant Physiology, Chinese Academy of \\ Sciences, 300 Fenglin Road, Shanghai 200032
}

\begin{abstract}
BcpCAL, the homologous gene of CAL, was isolated from Chinese cabbage. Unlike BobCAL of cauliflower, BcpCAL did not hold the terminating mutatzion in the fifth exon. After crosses of cauliflower with Chinese cabbage, the resultant hybrids failed to form curd, which implicates the genetic complement of BcpCAL to the mutated BobCAL in the function of curd formation. One of CAL gene isolated from the hybrid apparently comes from the female parent (Chinese cabbage) even though there are a few of the bases substituted and deleted. The result offers the molecular and genetic evidences for the study of biological function of CAL in morphological genetics of curd.
\end{abstract}

Key words: CAL gene, curd, Chinese cabbage, hybrid.

\section{INTRODUCTION}

The control of the transition from vegetative to reproductive development is being extensively studied in Arabidopsis thaliana. Genes determining the fate of lateral meristem initiated at the inflorescence meristem as floral meristem include CAULFLOWER (CAL), APETALA1 (AP1) and LEAFY (LFY)[1-3]. The predominant effect of mutations at the LEAFY locus or AP1 locus is to cause a large proportion of the lateral structures that ordinarily develop as flowers to be converted into inflorescence branches, or inter-

* The nucleotide sequence data reported in this paper will appear in DDBJ/EMBL/GenBank

Nucleotide Sequence Database under the accession number:AJ251300.

** Corresponding author. Fax: 086-021-64042090; E-mail: heyk@iris.sipp.ac.cn 
CAL gene in Chinese cabbage

mediate structures with characteristics of both flowers and branches[4,5]. When the ap1 mutation is combined with mutations in cal, cells that would normally constitute a floral meristem behave as an inflorescence meristem, giving rise to additional meristems in a spiral phylotaxy[3] (Bowmen, 1993). The resulting cauliflower phenotype has an extensive proliferation of meristems at each position. Genetic and molecular analyses reveal that the developmental function of CAL is partially redundant to AP1. It has been suggested that the molecular basis for the inflorescence structure in cauliflower (Brassica oleracea var. botrytis), which is similar to the cauliflower phenotype in Arabidopsis, is in part due to a stop codon mutation in the fifth exon5 of BobCAL gene[6]. To extend the knowledge of floral development further, we have focused on CAL genes and other related genes in Brassica species. Brassica is a complex species containing many varieties with grossly different phenotypes, including broccoli, cauliflower, Brussels sprouts, kale, cabbage and Chinese cabbage. Cauliflower phenotype produces an arrested development stage between the vegetative and floral differentiation, i. e. the characteristic edible white curd, while in broccoli phenotype development arrests at the later stage of immature floral buds.

In contrast, cabbage and Chinese cabbage phenotype produce leafy head that is a typical vegetative structure at the stage when curd appears in the case of cauliflower phenotype. Only after a vernalization period, plants begin to bolt and floral development ensues. Therefore, curd and leafy head become two special organs for investigating the difference between vegetative, inflorescence and floral meristems in which CAL, LFY and AP1 genes play a regulatory role. In order to correlate differences between cauliflower and Chinese cabbage in the formation of curd or leafy head, we have cloned and characterized the homolog of CAL genes from Chinese cabbage and F1 generation between Chinese cabbage and cauliflower.

\section{MATERIALS AND METHODS}

\section{Plant materials and growth conditions}

Seeds of cauliflower (Brassica oleracea var. botrytis) H6 and Chinese cabbage (Brassica campestris ssp. pekinensis) TT508 were obtained from our lab. Plants were grown in a greenhouse. In order to render both of the varieties to flower at the same time, Chinese cabbage seeds were sowed in July and cauliflower seeds in October. When temperature fell below $15^{\circ} \mathrm{C}$, cauliflower plants were transfer to controlled room with $18 / 22^{\circ} \mathrm{C}$ and $16 \mathrm{~h}$ daylength regime.

Chinese cabbage variety TT508 was designated as female parent and cauliflower variety H6 as male parent. The flowers of Chinese cabbage were emasculated at flower bud stage and pollinated with pollens of cauliflower. In order to get seeds of such interspecific hybridization, the embryo rescue was performed. The ovaries were cut from female plants seven days after pollination and cutured on medium under aseptic conditions. The seeds were harvested when they were fully matured. The plants of F1 generation were prepared as above-mentioned for Chinese cabbage.

\section{Isolation of the BcpCAL gene}

Genomic DNA of Chinese cabbage was prepared from 2-week-old plants according to the method described by Porebski[7]. More than six pairs of degenerated primers that specify the 
character of CAL gene were designed according to the published sequences of AtCAL (A. thaliania), $\mathrm{B}^{\circ} \mathrm{CAL}$ (cabbage)[6], BoiCAL (broccoli)[8] and PCR was performed to amplify two overlapping DNA fragments, covering the entire coding region of the CAL gene. Three pairs of primers were used to produce DNA upstream fragment of while other three pairs of primers to get the downstream fragment. PCR conditions were as follows: $94^{\circ} \mathrm{C}$ for $1 \mathrm{~min}$, followed by 35 cycles of $94^{\circ} \mathrm{C}$ for $1.5 \mathrm{~min}, 60^{\circ} \mathrm{C}$ for $1 \mathrm{~min}$, and $72^{\circ} \mathrm{C}$ for $1.5 \mathrm{~min}$. A TAQ: Pfu polymerase mixture (Stratagene) was used to minimize nucleotide misincorporation. The PCR products recovered from low melting point gel and were filled with the Klenow Large Fragment of DNA polymerase I, and inserted in plasmid of pBluscript $\mathrm{KS}(+)$ that was previously digested with EcoRV.

F1 plants of Chinese cabbage crossed with cauliflower were selected for the isolation of CAL gene. The same primers and PCR procedures were used and the PCR products were cloned into pGEMT vectors.

DNA inserts of all clones were sequenced on an Applied Biosystem 373 Automated DNA Sequenser (ABI/Perkin-Elmer, Foster City, CA). The resultant sequences were evaluated using Sequencer (Geme Codes, Ann Arbor, MI) for contig assembly and sequence editing. Splice sites were determined by comparison with cDNA sequences of BobCAL[6] and BoiCAL[8], using the software program Net Plant Gene available at internet.

\section{RESULTS}

\section{Morphological characters of hybrids of Chinese cabbage with cauliflower}

After extensive effort of pollination and embryo rescue, some seeds of hybrids of Chinese cabbage and cauliflower were obtained. The hybrids (F1 generation) grew normally either in greenhouse or in field and all produced the rosette leave like the parents. After rosette stage, folding leave were differentiated at shoot apices of Chinese cabbage resulting in leafy head, and floral development ensued only after a vernalization period (Fig 1A), and nearly normal inflorescence appeared from the shoot apices of hybrids (Fig 1B), whereas meristems proliferated at shoot apices of cauliflower resulting in curd (Fig 1C). In other words, the hybrids did not produce curd like cauliflower.

\section{Cloning and characterization of the BcpCAL gene}

Genomic DNA derived from leaves of Chinese cabbage was used as template to amplify two overlapping fragments of CAL genes. One PCR product of $1.3 \mathrm{~kb}$ was cleared with primers of P1 [5' -TCTACGAGAAATGGGAAGG-3']/P2 [5'-GTCGATATATGGCGAGTCC$3^{\prime}$ ] that could give rise to upstream fragment, while four products ranging from $1.6 \mathrm{~kb}$ to 2 . $2 \mathrm{~kb}$ were determined with primers of P3 [5' -G(C/T)TCCAGACTCTCACGTC-3']/P4 [5'-CCA(T/C)TGACCAGTTCGT(T/C) TG-3'](Fig 2A). Other four pairs of primers did not work very well in that they produced smearing or unclear bands. After restriction digestion, the upstream fragment of $1.3 \mathrm{~kb}$ and downstream fragment of $2.2 \mathrm{~kb}$ were sequenced. Alignment of two DNA fragments with CAL genes of Arabidopsis, cauliflower and cabbage, the homologous sequences of CAL gene in Chinese cabbage were verified. $1.3 \mathrm{~kb}$ fragment begins from first ATG and ends at the beginning of K region; $2.2 \mathrm{~kb}$ fragment starts at the sequence adjacent to $\mathrm{K}$ region and finishes just after stop codon. The two fragments were fitted each other, giving rise to the complete sequence of CAL gene of Chinese 


\section{CAL gene in Chinese cabbage}

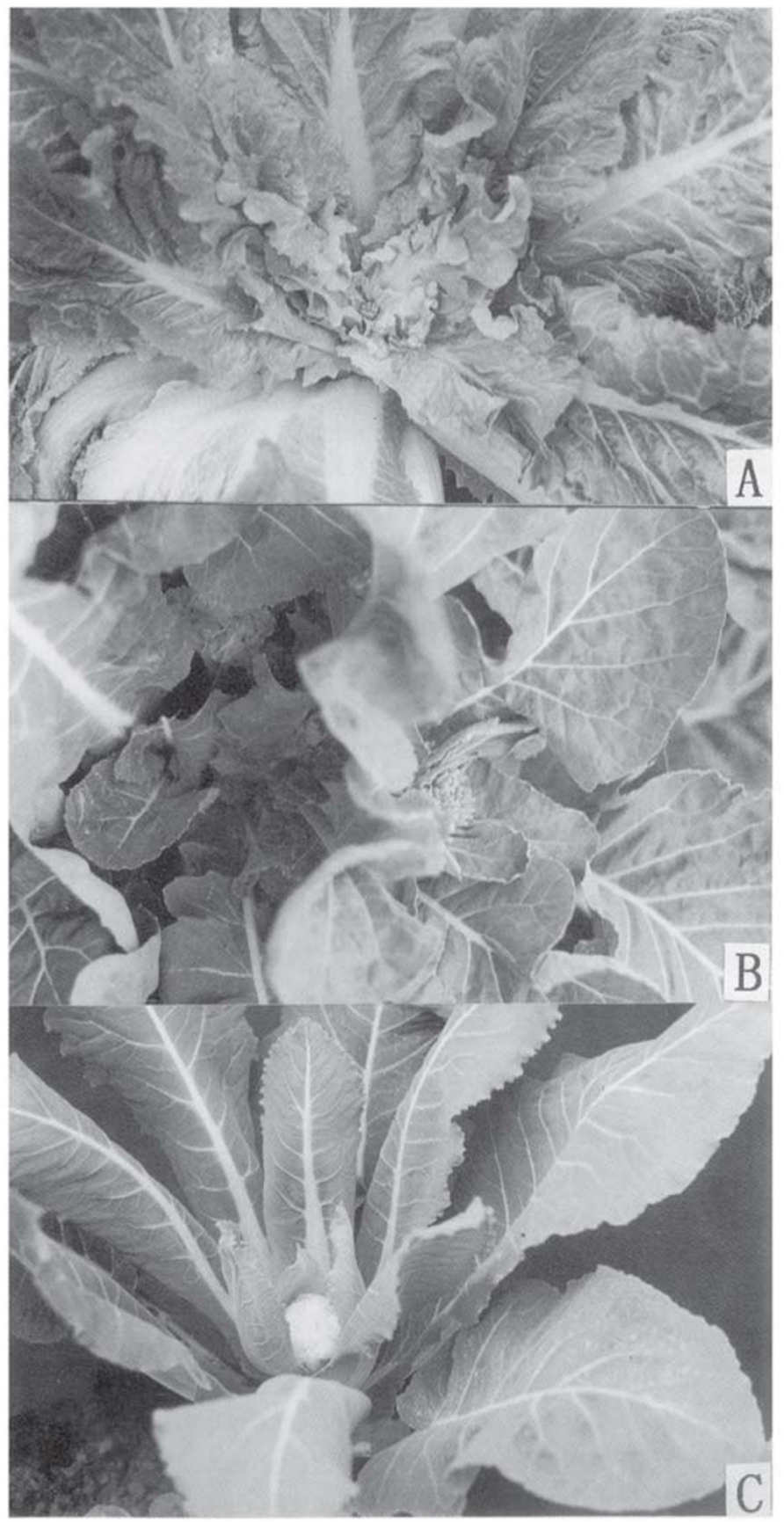

Fig 1.

Differences in developmental phenotypes at the beginning of inflorescence differentiation between Chinese cabbage, cauliflower and the hybrids of Chinese cabbage and cauliflower (F1 generation). A Chinese cabbage; B The hybrids of Chinese cabbage and cauliflower (F1 generation); C Cauliflower. 
A

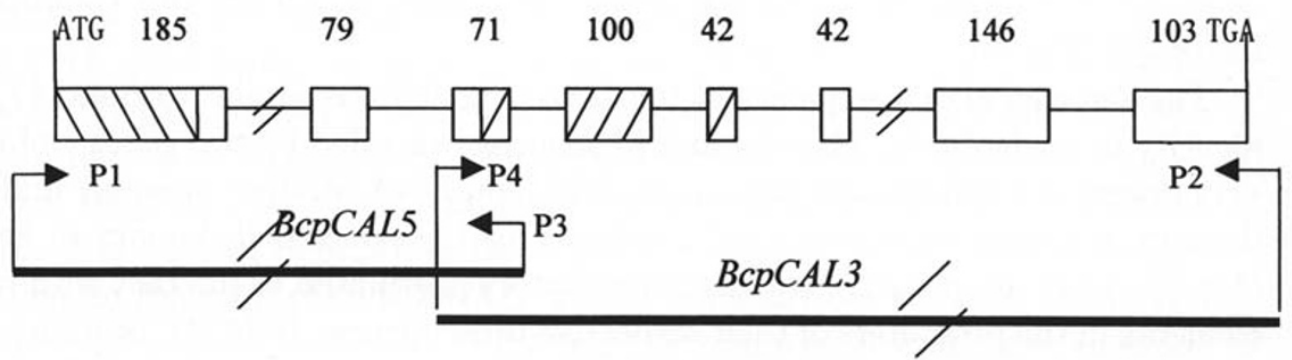

B

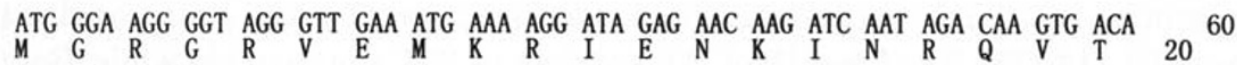

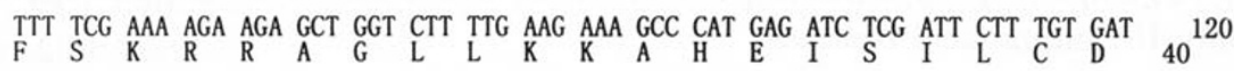

GCT GAG GTT TCC CTT ATT GTC TTC TCC CAT AAG GGG AAA CTG TTC GAG TAC TCG TCT GAA 180

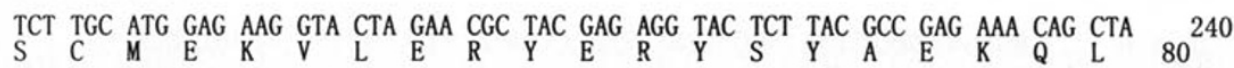

AAA GCT CCA GAC TCT CAC GTC AAT GCA CAA ACG AAC TGG TCA ATG GAA TAT AGC AGG CTT 300

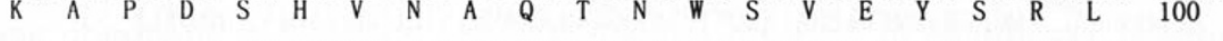

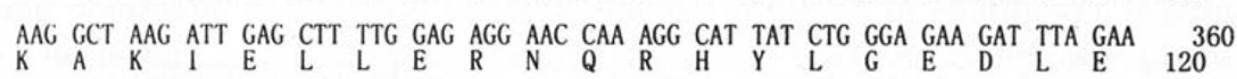

TCA ATC AGC ATA AAG GAG CTA CAG AAT CTG GAG CAG CAG CTT GAC ACT TCT CTT AAA CAT 420

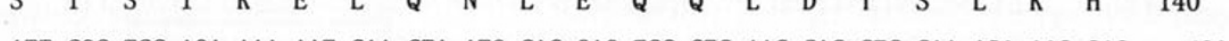

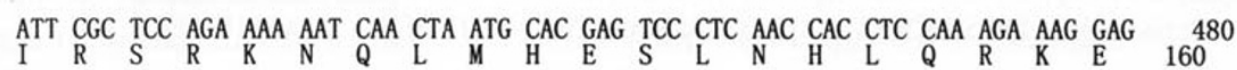

AAA GAA ATA CTT GAG GAA AAC AGC ATG CTT ACC AAA CAG ATA AAG GAG AGG GAG AGT ATC 540

K E. T L L E N S M L

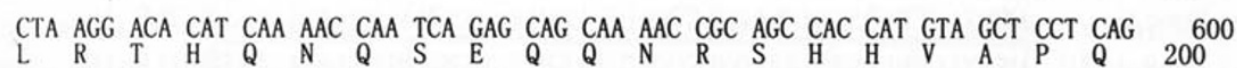

CCG CAG CCG CAG TTA AAT CCT TAC ATG ATC TCT CAT CAG GCA TCT CCT TTC CTA AGT ATG 660

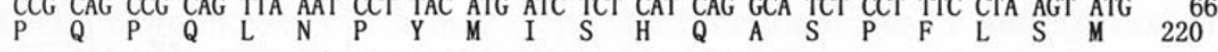

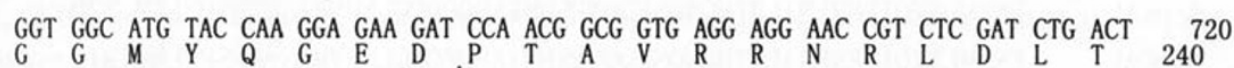

CTT GAA CCC ATT TAC AAC TGC AAC CTT GGT TAC TTT GCC GCA TGA

$254^{765}$

Fig 2.

The structure of BcpCAL gene. A. Exon-intron structure of BcpCAL gene and the position of primers designed. Exon s are shown as open boxes and introns as a solid line. Size (in base pairs) is indicated above. MADS- and K-boxes are hatched. Primers are represented by arrows, the PCR products of P1/P3 and P2/P4, BcpCAL5 and BcpCAL3, are indicated by bold solid line. B. Predicted cDNA and peptide sequence of BcpCAL. Single-letter abbreviations for the amino acid residues are as follows, and size (in base pairs and amino acid residues) is indicated on the right. Asterisk indicates the stop codon. A, Ala; C, Cys; D, Asp; E, Glu; F, Phe; G, Gly; H, His; I, Ile; K, Lys; L, Leu; M, Met; N, Asn; P, Pro; Q, Gln; R, Arg; S, Ser; T, Thr; V, Val; W, Trp;and Y, Tyr. 
CAL gene in Chinese cabbage

cabbage which is designated as $\mathrm{Bcp} C A L$. BcpCAL gene is highly homologous to $\mathrm{Bob} C A L$ of cauliflower, and each spans the length of $3.5 \mathrm{~kb}$ and contains seven introns[6] (Fig $2 \mathrm{~A})$.

The deduced cDNA sequence of $B c p C A L$ (Fig 2B) was similar to BobCAL $(90.2 \%$ identity in nucleotide). The amino acid sequence encoded by two genes embrace the very conserved MADS-box (56-amino acid-long DNA-binding domain) and the K-domain, a region with structural similarity to the coiled-coil domain in keratin[9] (Fig 3). They are putative transcription factors postulated to interact with response elements in the promoters of their respective target genes. BobCAL protein sequence of cauliflower is truncated due to a stop codon at position +151 downstream from start codon[6]. In the putative protein sequence of $B c p C A L$, there is no any stop codon around +151 aa, and instead there is an insertion of three amino acids at position $+212-214$ (Fig 3). Thus $B c p C A L$ consists of 254 amino acids while BobCAL has only 151 amino acid residues.

\begin{tabular}{|c|c|c|}
\hline cpCAL & MGRGRVEMKRIENKINRQVTFSKRRAGLLKKAHEISILCDAEVSLIVFSHKGKLF & 55 \\
\hline BobCAL & MGRGRVEMKRIENKINRQVTFSKRRAGLLKKAHEISILCDAEVSLIVFSHKGKLF & 55 \\
\hline BcpBobCAL & MGRGRVEMKRIENKINRQVTFSKRRAGLLKKAHEISILCDAEVSLIVFSHKGKLF & 55 \\
\hline BcpCAL & EYSSESCMELVLERYERYSYAEKQLKAPDSHVNAQTNWS VEYSRLKAKIELLERN & 110 \\
\hline BobCAL & EYSSESCMEKVLERYERYSYAEKQLKAPDSHVNAQTNWSMEYSRLKAKIELWERN & 110 \\
\hline BcpBobCAL & EYSSESCMEKVLERYERYSYAEKQLKAPDSHVNAQTNWS VEYSRLKAKIELLERN & 110 \\
\hline BcpCAL & QRHYLGEDLESISIKELQNLEQQLDTSLKHIRSRKNQLMHESLNHLQRKEKEILE & 16 \\
\hline BobCAL & QRHYLGEDLESISIKELQNLEQQLDTSLKHIRSRKNQLMH*SLNHLQRKEKEILE & 165 \\
\hline \multirow[t]{2}{*}{ BcpBobCAL } & QRHYLGEDLESISIKELQNLEQQLDTSLKHIRSRKNQLMHESLNHLQRKEKEILE & 165 \\
\hline & $\#$ & \\
\hline $\mathrm{cpCAL}$ & ENSMLTKQIKERESILRTHQNQSEQQNRSHHVAPQPQPQLNPYMISHQASPFLSM & 220 \\
\hline BobCAL & ENSMLAKQIKERESILRTHQNQSEQQNRSHHVAPQPQPQLNPYMAS--SPFLNM & 217 \\
\hline BcpBobCAL & ENSMLTKQIKERESILRTHQNQSEQQNRSHHVAPQPQPQLNPYMISHQASPFLNM & 220 \\
\hline BcpCAL & GGMYQGEDPTAVRRNRLDLTLEPIYNCNLGYFAA* & 254 \\
\hline ObCAL & GGMYQGEYPTAVRRNRLDLTLEPIYNCNLGYFAA* & 51 \\
\hline $\mathrm{cpBobC}$ & GGMYQGEDPTAVRRNRLDLTLEPIYNCNLGYFAA* & \\
\hline
\end{tabular}

Fig 3.

The alignment of deduced amino acid sequences of $B c p C A L, B o b C A L$ and $B c p B o b C A L$. The different amino acids are shaded, and the MADS-domain in bold and the K-domain boxed. Asterisks indicate stop codon and dash represents deletions. The different amino acids between $B c p C A L$ and $B c p B o b C A L$ are indicated by \# on them. The sequence after the first stop codon in BobCAL is underlined. 
One homolog of CAL gene in hybrids of Chinese cabbage crossed with cauliflower

F1 plants of Chinese cabbage crossed with cauliflower were selected for the isolation of $C A L$ gene using the same primers and PCR procedures. And the PCR products with length similar to that of Chinese cabbage were cloned and sequenced, which were named as $B c p B o b C A L$. This sequence was aligned to the sequences of two parents $B c p C A L$, $B o b C A L$ (Fig 4). Although the sequence of $B c p B o b C A L$ is highly homologous with $B c p C A L$ or BobCAL, it is different from either of them, but more similar to $B c p C A L$ than BobCAL. This is evident from the data shown in Fig 4A-C (Fig 4). Especially noticeable is the fact that there is no stop codon in exon5 of $B c p C A L$ as that in BobCAL (Fig 3). Therefore, the DNA sequence of $B c p B o b C A L$ apparently originates from the female parent that is Chinese cabbage. However, the substitution and deletion of base pairs which were found in the sequence indicates that DNA mutation has occurred in the interspecific hybrids of Chinese cabbage and cauliflower.

\section{DISCUSSION}

Curd formation of cauliflower is the natural event of morphological transformation from vegetative to arrested reproductive growth. Genetic studies suggest that there are one dominant and several recessive factors involved in cauliflower heading[10],[11]. The recent result of molecular studies shows that the cauliflower phenotype (curd) is due to the lack of a functional $C A L$ gene product[6]. However, the data pertaining to the mechanism of curd formation is limited and more evidences are needed to verify this morphological event from other species of Brassica.

Chinese cabbage, another member of Brassica genus, has the similar pattern of vegetative growth, but it produces leafy head instead of curd after rosette stage. Comparison of the sequence of $\mathrm{Bcp} C A L$ to that of $B o b C A L$ makes it clear that two sequences are identical except the single base substitution at the position of +151 in BobCAL of cauliflower which may cause the functional loss or malfunction of $C A L$ gene. This is in line with the suggested hypothesis of curd formation upon the loss- of -function[6].

The hybrids of Chinese cabbage and cauliflower failed to form curd, demonstrating that cauliflower phenotype is recessive. Since chromosomes of both Chinese cabbage and cauliflower were introduced into the hybrid, theoretically both $B c p C A L$ gene and $B o b C A L$ gene exist in the genome of the hybrids. This suggests that the functional factor(s) in Chinese cabbage is dominant to curd formation gene in cauliflower. If $B o b C A L$ is the main factor to interact with response elements involved in forming cauliflower phenotype, it implicates that $B c p C A L$ can be complementary to the mutant BobCAL in the curd phenotype. The molecular analyses revealed that the sequence of $B c p B o b C A L$ in $\mathrm{F} 1$ hybrids which we isolated is much more similar to that of $B c p C A L$ than $B o b C A L$, especially there is no stop codon in fifth exon like that in $B c p C A L$, a fact which suggests that $B c p B o b C A L$ comes from $B c p C A L$. These data indicate that one of $C A L$ homolog in hybrids 
CAL gene in Chinese cabbage

$\mathbf{A}$

BcPCAL TCTTTTCOGTAATAAAAGTTAATGT.TTAGATGCATCGCTAATTAATTAGGTAAACTAGA

BcpBobCAL TCTTTTCOGGTATAAAAGTTAATGT. TTAG GTGCATCGCTAATTAATTAGG IAAACTAGA

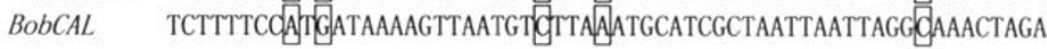

BCPCAL TGATATAAA........... TGTGTGTGTATTGGATATTTTGGGTTAATAGTTACATCT

BcpBobCAL TGATATAA ............ TGTGTGTGTATTGGATATTTTGGGTTAATAGTTACATCT

BobCAL TGATAGTACGTAGTGTGTGTGTGTGTGTGTATTGGATATTTTGGGTAATAGTTACATCT

B

BcPCAL GTAAGTAATAAGCACATACAAACGCAA .........TCTTATCTTTAAGTTTATGAAGD

BcpBobCAL GTAAGTAAATAAGCACATACAAACGCAA ......... TCTTATCTTTAAGTTTATGAAG

BObCAL GTGTGTAAATAAGCACATACAAACGCAAACATCTCTATCTTATCTTTGAGTTTGTGAAGA

BcpCAL TATATATGCCTAATTTTITATATAGAGTTTGTCTCATATGAATGAATACAATTOGGGACTCA

BcpBobCAL TATATATGCCTAATTTT. ATATAGAGTTTGTCTCATATGAATGAATACAATTOGGG̈ACTCA

BObCAL TATATATGCCTAATTTT. ATATAGAGTTTGTCTCATATGAATGAATACAATTIIGÄACTCA

C

BcpCAL AATCAACTAATGCAOGAGTCCCTCAADCACCTCCAAAGAAAGGTACGTTAAAACCATTTC

BcpBObCAL AATCAACTAATGCAOGAGTCCCTCAAIICACCTNCAAAGAAAGGTOCGTTAAAACCATTTC

BObCAL AATCAACTAATGCACTAGTCCCTCAADCACCTCCAAAGAAAGGTAGCGTTAAAACCATTTC

BCPCAL ATCTCTCAAGTCGTACGTGTGTATGTGTGACTTATGTTACCGTTTAAATCTTTCAGTTAT

BcpBobCAL ATCTCTCAAGTCGNACGTGTGTATGTGTGACTTATGTT. CCGTT. AAATCTTTCAGTTAT

BobCAL ATCTCTCAAGTCGTACGTGTGTATGTGTGACTTATGTTÄCCGTTIAAATCTTTCAGTTA.

BcPCAL GTTACAAAAAAAAATCTTTCAGTTAAATACAAAACATATGGTTTTACACATGTTAGACTA

BcpBobCAL GT. ACAAAAAAAA. TCTTTCAATTAAAT. CAAAACATATGGTTTTACACATGTTANACTA

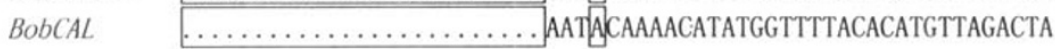

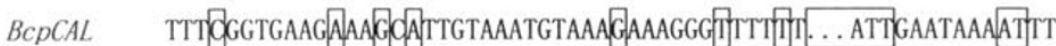

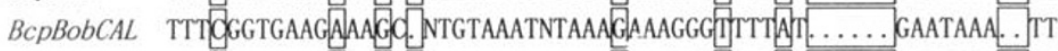

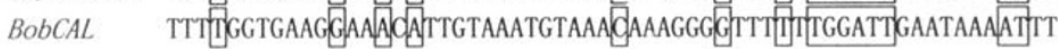

BCPCAL AACATICATTITAAAAAGACATATGGITCATATATAT. . TCGGTTTATATGATTATATCGT

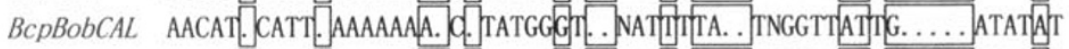

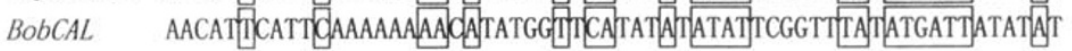

Fig 4.

Alignment of partial genomic DNA sequences to show the differences between BcpCAL $B o b C A L$ and $B c p B o b C A L$ genes. A. One internal fragment of the first intron; B. One internal fragment of the forth intron. C. The fifth exon and start of the fifth intron. The exon is shaded and the different bases are boxed, and dots represent deletions. 
isolated by us comes from maternal gene, but the progress of crossing may change the molecular sequence. In addition, most molecular variation are localized in introns of which the function is not known.

On the other hand, cauliflower genes can be complementary to the genes modifying leafy head phenotype in Chinese cabbage. It is generally known that much of plant development occurs through the reiterative production of organ primordia at the shoot apical meristem (SAM) and the developmental fate of primordia initiated at the SAM is controlled by environmental and endogenous signals[14],[15]. Both structures of leafy head and curd could result from the proliferation of lateral primordia, in which if the primordium enters into the fate of leave program becoming leafy head like that in Chinese cabbage, and if it arrests at the developmental stage between the vegetative form and floral differentiation giving bumpy white curd like that in cauliflower. In addition, both structures are due to the lack of internode elongation. So genes involved in controlling the formation of curd and leafy head include not only the meristem identity genes such as $L F Y, A P 1$ and $C A L$, but also the genes controlling internode elongation and lateral meristem number which are not yet known. It has been suggested that mutant $C A L$ alleles are associated with an increase in the number of axillary inflorescence[3]. So how $C A L$ gene functions in the development of Brassicae species and what is its upstream and downstream genes of this control point need to be further studied and elaborated. Generally, in hybrids, the normal expression of $B c p C A L$ gene could complement the lost function of BobCAL gene, leading to the recessiveness of cauliflower phenotype.

It should be noticed that the base substitution and deletion happen in the genomic sequence of $B c p B o b C A L$ gene deriving from $B c p C A L$ gene of Chinese cabbage. Apparently, there is DNA mutation in genome of the hybrids that is not clear about how and when it will happen. It is also not clear whether such limited mutation may change the expression level or biological function of $C A L$ gene. However the way in which $C A L$ gene takes mutation may throw light in evolutionary event.

In Arabidopsis, double mutations of $C A L$ and $A P 1$ genes cause the cauliflower phenotype[3], [12],[13]. We found that cauliflower, upon breaking arrest, produce typical cruciform flowers which do not display any homeotic transformation as in the double mutants of Arabidopsis with cal and ap1. genes. These results suggest that the progress of curd formation of our hybrids may be different from that of the heading phenotype in double mutant of Arabidopsis.

\section{ACKNOWLEDGEMENTS}

We would like to thank Prof. Xu Zhihong of Shanghai Institute of Plant Physiology, the Chinese Academy of Sciences, for his comment and criticism. This work was supported by National Climbing Project (95-29-06). 


\section{CAL gene in Chinese cabbage}

\section{REFERENCES}

[1] Schulta EA and Haughn GW. LEAFY, a homeotic gene that regulates inflorescence development in Arabidopsis. The Plant Cell 1991; 3:771-81.

[2] Weigel D, Alvarez J, Smyth DR, Yanofsky MF and Meyerowitz EM. LEAFY controls floral meristem identity in Arabidopsis. Cell 1992; 69:843-59.

[3] Bowman JL, Alvarez J, Weigel D, Meyerowitz EM and Smyth DR. Control of flower development in Arabidopsis thaliana by APETALA1 and interacting genes. Development 1993; 119:721-43.

[4] Huala E and Susses IM. LEAFY interacts with floral homeotic genes to regulate Arabidopsis floral development. The Plant Cell 1992; 4:901-13.

[5] Ratcliffe OJ, Bradley DJ and Coen ES. Separation of shoot and floral identity in Arabidopsis. Development 1999; 121:1109-20.

[6] Kempin SA, Savidge B and Yanofsky MF. Molecular basis of the cauliflower phenotype in Arabidopsis. Science 1995; 267:522-5.

[7] Porebski S, Bailey LG and Baum BR, Modification of a CTAB DNA extraction protocol for plants containing high polysaccharide and polyphenol components, Plant Molecular Biology Reporter 1997; 15(1):8-15.

[8] Susan MC and Irish VF, Floral homeotic gene expression defines developmental arrest stages in Brassica oleracea L. vars. botrytis and italica, Planta 1997; 201:179-88.

[9] Ma H, Yanofsky MF and Meyerowitz EM. AGL1-AGL6 an Arabidopsis gene family with similarity to floral homeotic and transcription factor genes. Genes Dev 1991; 5:484-95.

[10] Pease MS. Genetic studies in Brassica oleracea. J Gen 1926; 16:363-85.

[11] Crisp P. The use of an evolutionary scheme for cauliflowers in the screening of genetic resources. Euphytica 1982; 31:1311-22.

[12] Anthony RG, James PE and Jordan BR. The cDNA sequence of a cauliflower apetala- 1/squamosa homolog. Plant Physiol 1995; 108:441-2.

[13] Anthony RG, James PE and Jordan BR. Cauliflower (Brassica oleracea var. botrytis L.) curd development: the expression of meristem identity genes. J Exp Bot 1996; 47:181-8.

[14] Bernier G. The control of floral evocation and morphogenesis. Annu.Rev. Plant Physiol. Plant Mol Biol 1988; 39:175-219.

[15] McDaniel, CN, Singer SR and Smith SME. Developmental states associated with the floral transition. Dev Biol 1992; 153:59-69

\section{Received Nov-24-1999. $\quad$ Revised Jan-26-2000. Accepted Jan-28-2000.}

